\title{
IMEX-MCNAB Scheme for Pricing European Options under Kou's Jump- Diffusion Models
}

\author{
Xiangyu Jia, Zuoliang Xu \\ School of Information, Renmin University of China Beijing 100872, P. R. China \\ ajxy_dbw@ruc.edu.cn, bxuzl@ruc.edu.cn
}

Keywords: implicit-explicit methods; linear multistep methods; jump-diffusion model; option pricing; Fourier stability analysis

\begin{abstract}
We consider IMEX-MCNAB time discretization scheme for the partial integrodifferential equation derived for the pricing of options under a jump-diffusion process. The scheme is defined by a convex combination parameter, which divides the zeroth-order term due to the jumps between the implicit and explicit parts in the time discretization. This scheme is studied through Fourier stability analysis. It is found that, under suitable assumptions and time step restrictions, the IMEX-MCNAB scheme is conditionally stable. Numerical experiments show the effectiveness of the proposed method.
\end{abstract}

\section{Introduction}

Options, as an important financial derivative, are very active in the current financial market. In 1973, F. Black and M. Scholes ${ }^{[1]}$ proposed the famous Black-Scholes model. But the Black-Scholes model, despite its simplicity, ignores some of market features, such as market jumps and crashes, whereas these are such important events that should be considered.

In 1976, R. Merton ${ }^{[2]}$ proposed the addition of jumps into the Black-Scholes model. Contrary to models with continuous paths, jump-diffusion models allow large sudden changes in the price of the underlying asset. In ${ }^{[3]}$, S.G. Kou introduced a jump-diffusion model in which amount of jump has a distribution of log-double exponential type. In a richer CGMY model ${ }^{[4]}$, the asset price is a Levy process with possibly infinite jump activity.

A solution to a jump-diffusion model can be obtained by solving a partial integro-differential equation (PIDE). Direct numerical issues were already considered in ${ }^{[5,6]}$. However, direct solution methods are usually too expensive with a full matrix, and therefore other numerical methods should be considered. D. Tavella and C. Randall ${ }^{[7]}$ described a stationary iterative method for pricing European options. S. Salmi, J. Toivanen ${ }^{[8]}$ proposed an iterative method for pricing American options under jump-diffusion models.

U. Ascher, S. Ruuth and B. Wetton ${ }^{[9]}$ introduced implicit-explicit methods for time-dependent partial differential equations including the IMEX-MCNAB scheme. J. Frank, W. Hundsdorfer and J. Verwer ${ }^{[10]}$ analyzed the stability of implicit-explicit linear multistep methods. S. Salmi, J. Toivanen [11] discussed some IMEX-schemes for pricing options under jump-diffusion models.

In this paper, we discuss IMEX-MCNAB scheme for pricing European options under Kou's jumpdiffusion model. We also put a convex combination of the zeroth-order term into the IMEXMCNAB scheme which was introduced in ${ }^{[11]}$. Then this scheme is studied with Fourier stability analysis and numerical experiments show the effectiveness of the proposed method.

This paper is organized as follows. Section 1 consists of the introduction. In Section 2, Kou's models for European options under jump-diffusion processes are introduced. In section 3, we consider the IMEX-MCNAB scheme and study its stability through Fourier analysis. In section 4, numerical experiments show the effectiveness of the proposed method. Finally, this paper concludes with a conclusion section. 


\section{Kou's Models for European options}

Under the risk neutral measure, the underlying asset price $x$ is governed by the following stochastic differential equation

$$
\frac{d x}{x}=(\mu-\lambda \kappa) d t+\sigma d W+(\eta-1) d q
$$

where $\mu$ is the drift rate, $\sigma$ is the volatility, $d W$ is an increment of Gauss-Wiener process, $\eta-1$ is an impulse function producing a jump from $x$ to $\eta x, \kappa=E[\eta-1]$, where $E[\cdot]$ denotes the expectation operator, and $d q$ is the independent Poisson process with arrival rate $\lambda$.

Now suppose $v(x, t)$ as the price of European option with underlying asset model of (1), then $v(x, t)$ satisfies the following PIDE

$$
\begin{aligned}
& v_{\tau}=L v:=\frac{1}{2} \sigma^{2} x^{2} v_{x x}+(r-\lambda \kappa) x v_{x} \\
& -(r+\lambda) v+\lambda \int_{0}^{\infty} v(x \eta, \tau) g(\eta) d \eta,
\end{aligned}
$$

where $\tau$ is the time to maturity, $x \in[0, \infty), \tau \in[0, T)$, and $T$ is the maturity, $r$ is the risk-free interest rate, and $g(\eta)$ is the probability density function of the jump amplitude $\eta$ satisfying $\int_{0}^{\infty} g(\eta) d \eta=1$. In this paper, we consider Kou's model.

In Kou's model, $g(\eta)$ is following log-double-exponential density

$$
g(\eta)=p \eta_{1} \exp \left(-\eta_{1} \eta\right) H(\eta)+q \eta_{2} \exp \left(\eta_{2} \eta\right) H(-\eta)
$$

where $\eta_{1}>1, \eta_{2}, p, q>0, p+q=1$, and $H(\eta)$ is the Heaviside function. It can be shown that, in this case

$$
\kappa=E[\eta-1]=\frac{p \eta_{1}}{\eta_{1}-1}+\frac{q \eta_{2}}{\eta_{2}-1}-1
$$

In the case of an European put option, the initial and boundary conditions are

$$
\left\{\begin{array}{l}
v(x, T)=(K-x)^{+} \\
v(0, \tau)=K e^{-r \tau} \\
\lim _{x \rightarrow \infty} v(x, \tau) \rightarrow 0
\end{array}\right.
$$

To simplify notation, we split the PIDE (2) into parts as follows

$$
v_{\tau}=L v=D v+\lambda J v-\lambda v
$$

where $D$ is the differential operator and $J$ is the integral operator defined by

$$
\left\{\begin{array}{l}
D v:=\frac{1}{2} \sigma^{2} x^{2} v_{x x}+(r-\lambda \kappa) x v_{x}-r v \\
J v:=\int_{0}^{\infty} v(x \eta, \tau) g(\eta) d \eta
\end{array}\right.
$$

\section{Time stepping scheme and stability}

In the following, we include the extra parameter $c \in[0,1]$, for the convex combination of the zeroth-order term $\lambda v$ between the differential part and integral part of (4)

$$
v_{\tau}=L v=(D-\lambda(1-c) I) v+\lambda(J-c I) v,
$$

where $I$ is the identical operator.

We consider the stability properties of IMEX-MCNAB (implicit-explicit, modified CrankNicolson, Adams-Bsahforth) time-stepping scheme of (3.1), which is 


$$
\begin{aligned}
& \boldsymbol{v}_{m+1}-\boldsymbol{v}_{m}=\Delta \tau \lambda(\boldsymbol{J}-c \boldsymbol{I})\left(\frac{3}{2} \boldsymbol{v}_{m}-\frac{1}{2} \boldsymbol{v}_{m-1}\right)+ \\
& \Delta \tau(\boldsymbol{D}-\lambda(1-c) \boldsymbol{I})\left(\frac{9}{16} \boldsymbol{v}_{m+1}+\frac{3}{8} \boldsymbol{v}_{m}+\frac{1}{16} \boldsymbol{v}_{m-1}\right),
\end{aligned}
$$

where $\boldsymbol{v}_{m}=\boldsymbol{v}(m \Delta \tau), \boldsymbol{I}$ is the identity matrix, $\boldsymbol{D}$ and $\boldsymbol{J}$ are matrices resulting from the spatial discretization of (5). This scheme was previously studied in a more general context in [9]. The extra parameter $c \in[0,1]$ for the convex combination of the zeroth-order term $\lambda v$ leads to a family of methods for the IMEX-MCNAB scheme, with a particular method defined by the value $c$. We adopt the finite difference method for the spatial discretization, see [8] for details on the discretization.

A scheme is conditionally stable if it can be shown to be stable for $\Delta \tau$ smaller than a given positive value. This is a more stringent requirement, and zero stability clearly follows from conditional stability. Unfortunately, the stability region of a scheme applied to a general problem is often difficult to prove rigorously. The usual approach is to apply the scheme to the linear test problem and analyze its stability. In the case of IMEX-schemes, the linear test equation reads

$$
v^{\prime}(\tau)=v_{B} v(\tau)+v_{C} v(\tau)
$$

where $v_{B}$ and $v_{C}$ are the complex eigenvalues of the explicit and implicit part of the scheme, respectively.

By applying the so-called method of lines approach on the PIDE (2), we obtain a semi-discrete linear system of ODEs

$$
\boldsymbol{v}^{\prime}(\tau)=\boldsymbol{D} \boldsymbol{v}(\tau)+\boldsymbol{J} \boldsymbol{v}(\tau)-\lambda \boldsymbol{v}(\tau), \quad \tau \geq 0
$$

Stability results for the test equation (8) can be readily extended to linear systems with commuting matrices. The stability of IMEX-schemes in a commutative framework was discussed in [10], for example. However, above $\boldsymbol{D}$ and $\boldsymbol{J}$ do not commute in general. Nevertheless, in many practical applications it has been found that time step size restrictions based on the linear test equation are accurate even in the noncommutative case [12]. In the following, we will analyze the stability of the IMEX-MCNAB scheme (7) applied to the linear test equation (8).

\subsection{Stability of the test problem}

The stability of an IMEX linear multistep method, as in [10], is determined by the roots of the characteristic equation

$$
\sum_{i=0}^{l} a_{i} \zeta^{l-i}=v_{B} \sum_{i=1}^{l} b_{i} \zeta^{l-i}+v_{C} \sum_{i=0}^{l} c_{i} \zeta^{l-i}
$$

where $a_{i}, b_{i}$ and $c_{i}$ are the coefficients that define the particular IMEX-scheme, as in relation (1.2) in [10], and where $l$ is the number of past steps involved in the scheme. The scheme is stable if all roots satisfy $|\zeta| \leq 1$, with strict inequality for multiple roots. We adopt the formulation in [10] by dividing the equation by $\zeta^{l}$ and substituting $z=1 / \zeta$. Now the characteristic equation reads

$$
A(z)=v_{B} B(z)+v_{C} C(z),
$$

where $A, B$ and $C$ are given by

$$
A(z)=\sum_{i=0}^{l} a_{i} z^{i}, \quad B(z)=\sum_{i=1}^{l} b_{i} z^{i}, C(z)=\sum_{i=0}^{l} c_{i} z^{i}
$$

Stability holds if all roots satisfy $|z| \geq 1$, again with strict inequality if $z$ is a multiple root. A necessary condition for this is

$A(z)-v_{B} B(z)-v_{C} C(z) \neq 0$, for all $|z|<1$. (3.8)

Apart from the possibility of multiple roots of modulus 1 this is also a sufficient condition.

For the IMEX-MCNAB scheme (7), the polynomials in (11) are given by 


$$
A(z)=1-z, B(z)=\frac{3}{2} z-\frac{1}{2} z^{2}, C(z)=\frac{1}{16}(3+z)^{2},
$$

where $v_{B}$ and $v_{C}$ are the eigenvalues of

$$
\left\{\begin{array}{l}
\Delta \tau \lambda(\boldsymbol{J}-c \boldsymbol{I}) \boldsymbol{v}=v_{B} \boldsymbol{v}, \\
\Delta \tau(\boldsymbol{D}-\lambda(1-c) \boldsymbol{I}) \boldsymbol{v}=v_{C} \boldsymbol{v} .
\end{array}\right.
$$

Let the eigenvalues $v_{\boldsymbol{J}}$ and $v_{\boldsymbol{D}}$ be given by

$$
J v=v_{J} v, \quad D v=v_{D} v
$$

Then it holds

$$
v_{B}=\Delta \tau \lambda\left(v_{J}-c\right), v_{C}=\Delta \tau\left[v_{D}-\lambda(1-c)\right] .
$$

Substituting $v_{B}$ and $v_{C}$ in (16) into the characteristic polynomial gives

$$
1-z=\Delta \tau \lambda\left(v_{J}-c\right) \frac{3 z-z^{2}}{2}+\Delta \tau\left[v_{D}-\lambda(1-c)\right] \frac{(3+z)^{2}}{16} .
$$

Solving $v_{J}$ from the above equation yields

$$
v_{J}=c+\frac{2}{3 z-z^{2}}\left[\frac{1}{\lambda \Delta \tau}(1-z)+\frac{1}{16}\left(1-c-\frac{v_{D}}{\lambda}\right)(3+z)^{2}\right] .
$$

The boundary of the stability region for $v_{J}$ is obtained by setting $z=e^{i \theta}$, substituting this into (18), and letting $\theta_{\text {vary in the range }}[0,2 \pi]$. Stability boundaries for the IMEX-MCNAB scheme are plotted in Figure 1 for $v_{D}=0$. The green unit ball illustrates the area of possible eigenvalues $\left|v_{J}\right| \leq 1$. This suggests that the IMEX-MCNAB scheme becomes stable for all $c \in[0,1]$ once $\lambda \Delta \tau<1 / 2$, while for $c=0$ the stability condition becomes even more relaxed at $\lambda \Delta \tau<1$.
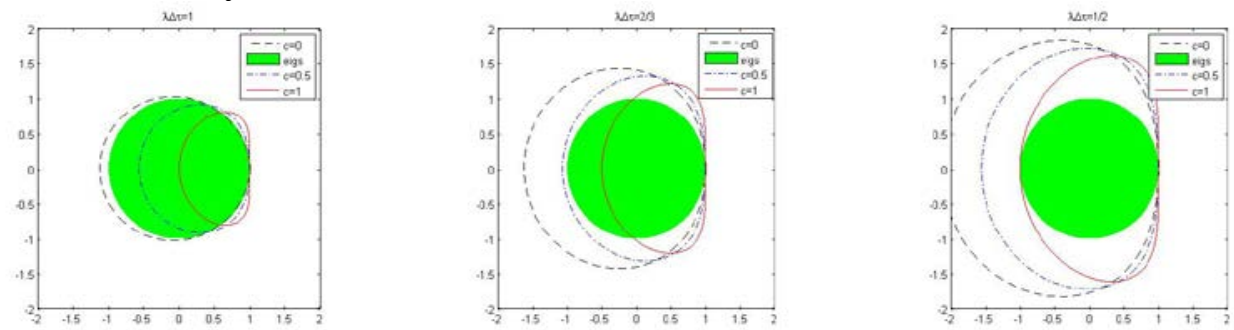

Figure 1. Stablity boundaried for $\lambda \Delta \tau=1$ (left), $\lambda \Delta \tau=2 / 3$ (center) and $\lambda \Delta \tau=1 / 2$ (right) with

$$
v_{D}=0 \text {. }
$$

We will now formally investigate the stability regions of the IMEX-MCNAB scheme. We will assume that $\boldsymbol{J}$ does not have error due to the truncation of the domain. This leads to a non-negative right stochastic matrix $\boldsymbol{J}$. An alternative path is to assume that $\boldsymbol{J}$ has truncation error and is nonnegative. In this case $\left|v_{J}\right|<1$ holds due to the Gershgorin circle theorem. Thus, roots of modulus 1 do not exist, and the theoretical results below would be valid even if $\boldsymbol{J}$ is not right stochastic. We assume that $\boldsymbol{J}$ does not have truncation error because it is a more general framework.

In [11], we have the following lemma.

Lemma 1. If $\boldsymbol{J}$ is strictly positive, then $\left|v_{\boldsymbol{J}}\right| \leq 1$ and roots of modulus 1 are simple.

Proposition 1. If $\boldsymbol{J}$ is strictly positive, then for real $v_{\boldsymbol{D}} \leq 0$ the IMEX-MCNAB scheme is conditionally stable for all $\lambda \Delta \tau<1 / 2$ and $c \in[0,1]$.

Proof. We know that the characteristic equation is satisfied for

$$
v_{J}=c+\frac{2}{3 z-z^{2}}\left[\frac{1}{\lambda \Delta \tau}(1-z)+\frac{1}{16}\left(1-c-\frac{v_{D}}{\lambda}\right)(3+z)^{2}\right] \text {. }
$$


From Lemma 1, it shows that $\left|v_{J}\right| \leq 1$. Assume that $|z|<1$ and that equation (19) holds. In the following we will show that this will lead to a contradiction. Denote $k=1 / \lambda \Delta \tau>2$ and $z=a+b i(-1<a<1,1<b<1)$.

Multiplying equation (19) by $z$ yields

$$
\begin{aligned}
& v_{J} \cdot z=c z+\frac{2}{3-z}\left[k(1-z)+\frac{1}{16}\left(1-c-\frac{v_{D}}{\lambda}\right)(3+z)^{2}\right] \\
& =a c+b c i+2 k-\frac{1}{8}\left(1-c-\frac{v_{D}}{\lambda}\right)(9+a+b i) \\
& +\left[\frac{9}{2}\left(1-c-\frac{v_{D}}{\lambda}\right)-4 k\right] \frac{(3-a)+b i}{(3-a)^{2}+b^{2}} .
\end{aligned}
$$

Now we can show that $\operatorname{Re}\left(v_{J} \cdot z\right) \geq 1$, which means $\left|v_{J} \cdot z\right| \geq 1$.

$$
\begin{aligned}
& \operatorname{Re}\left(v_{J} \cdot z\right)=a c+2 k-\frac{1}{8}\left(1-c-\frac{v_{D}}{\lambda}\right)(9+a) \\
& +\left[\frac{9}{2}\left(1-c-\frac{v_{D}}{\lambda}\right)-4 k\right] \frac{(3-a)}{(3-a)^{2}+b^{2}} .
\end{aligned}
$$

Then

Thus, it holds

$$
\frac{d R e\left(v_{J} \cdot z\right)}{d k}=2-\frac{4(3-a)}{(3-a)^{2}+b^{2}} \geq 2-\frac{4}{3-a}>0
$$

$$
\begin{aligned}
& \operatorname{Re}\left(v_{J} \cdot z\right)>a c+4-8 \frac{(3-a)}{(3-a)^{2}+b^{2}} \\
& +\left(1-c-\frac{v_{D}}{\lambda}\right)\left[\frac{9}{2} \frac{(3-a)}{(3-a)^{2}+b^{2}}-\frac{a+9}{8}\right] .
\end{aligned}
$$

Let $M=\frac{9}{2} \frac{(3-a)}{(3-a)^{2}+b^{2}}-\frac{a+9}{8}$, then we have

$$
M>\frac{-a^{3}-3 a^{2}+8 a+18}{8\left[(3-a)^{2}+1\right]} .
$$

Denote $f(a)=a^{3}+3 a^{2}-8 a-18$, through analyzing the first and second derivative of it, we can conclude that the maximum value of $f(a)$ in the interval $(-1,1)$ is obtained at the two endpoints $a= \pm 1$, and $f(-1)=-11<0, f(1)=-22<0$. Thus $f(a)$ is negative in the interval $(-1,1)$, which leads $M>0$, and the next inequalities hold

$$
\operatorname{Re}\left(v_{J} \cdot z\right)>a c+4-\left(\frac{7}{2}+\frac{9}{2} c\right) \frac{(3-a)}{(3-a)^{2}+b^{2}}-\frac{a+9}{8}(1-c)>\left(a-\frac{9}{2} \frac{1}{3-a}+\frac{a+9}{8}\right) c+4-\frac{7}{2} \frac{1}{3-a}-\frac{a+9}{8} .
$$

In the above expression, denote $N=a-\frac{9}{2} \frac{1}{3-a}+\frac{a+9}{8}$ which is the coefficient of $c$, it holds

$$
N=\frac{-9(a-1)^{2}}{8(3-a)}<0
$$

So the expression is minimized by the choice $c=1$, and the next inequality holds

So we have

$$
\operatorname{Re}\left(v_{J} \cdot z\right)>4+a-\frac{8}{3-a} \text {. }
$$




$$
\operatorname{Re}\left(v_{J} \cdot z\right)-1>3+a-\frac{8}{3-a}=\frac{1-a^{2}}{3-a}>0 .
$$

The above analysis shows that $\operatorname{Re}\left(v_{J} \cdot z\right)>1$, and thus $\left|v_{J} \cdot z\right|>1$. This leads to $\left|v_{J}\right||z|>1$, and also $\left|v_{J}\right| \geq\left|\frac{1}{z}\right|>1$, which is a contradiction with Lemma 1. Hence, there is no $|z|<1$ suct holds, which means that the necessary condition for stability (3.8) holds. This is also sufficient, since Lemma 1 guarantees that roots of modulus 1 are simple.

Under Kou's models, $\boldsymbol{J}$ is strictly positive for any quadrature with positive weights. We can obtain the following corollary from Propositions 1.

Corollary 1. If $v_{D} \leq 0$, then the IMEX-MCNAB scheme is conditionally stable under Kou's models for all $\lambda \Delta \tau<1 / 2$ and $c \in[0,1]$.

\subsection{Stability of the full problem}

In the analysis, we assumed that $v_{D} \leq 0$. A sufficient condition for the eigenvalue $v_{D}$ being real and nonpositive is, for example, that $\boldsymbol{D}$ is tridiagonal and M-matrix. Under $r \geq 0$, the M-matrix property can be attained by adding artificial diffusion into the model, as was done in [13] for example.
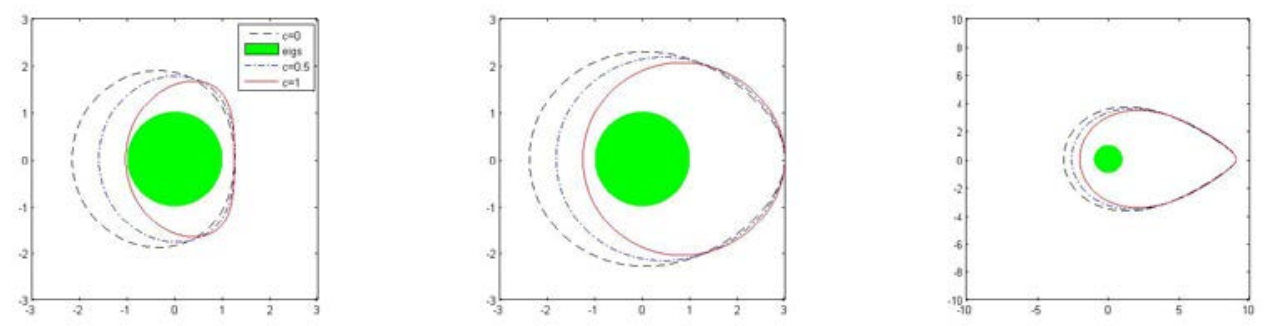

Figure 2. Stablity boundaried for $v_{D}=-1 / 8$ (left), $v_{D}=-1$ (center) and $v_{D}=-4$ (right) with $\lambda \Delta \tau=1 / 2$.

The formal connection between the stability of the linear test problem and the system of ODEs (9) holds, for example, in the special case $v_{\boldsymbol{D}}=0$. Stability regions for $v_{\boldsymbol{D}}=-1 / 8, v_{\boldsymbol{D}}=-1$, and $v_{\boldsymbol{D}}=-4$ are illustrated in Figure 2 for the IMEX-MCNAB scheme. The stability region improves for any real $v_{D}<0$. Thus, under these assumptions, the stability of the scheme only improves with negative $v_{D}$. This suggests that under suitable assumptions, the special case $v_{D}=0$ can be considered as the worst case stability scenario.

\section{Numerical experiments}

In this section, we present numerical results computed with the IMEX-MCNAB scheme. We price European put options under Kou's models using the following model parameters

$$
\begin{aligned}
\sigma & =0.15, r=0.05, T=0.25, K=100, \\
\lambda=0.1, \alpha_{1} & =3.0456, \alpha_{2}=3.0775, p=0.3445 .
\end{aligned}
$$

These parameters are also used by d'Halluin, Forsyth and Vetzal in [6] and Toivanen in [13]. We use the reference prices described in [13] as follows

TABLE I. Reference price used for numerical experiments

\begin{tabular}{c|rcc}
\hline Model & Value at 90 & Value at 100 & Value at 110 \\
\hline Kou & 9.430457 & 2.731259 & 0.552363 \\
\hline
\end{tabular}

A uniform space grid between $[0, X]$ is used with $n$ nodes and $X=400$. We continue the 
iteration until $\left\|v^{l}-v^{l+1}\right\|<10^{-8}$. The root mean square errors (RMSE) were computed at spatial nodes $x_{i}=\{90,100,110\} . \quad R M S E=\sqrt{\left[\left(v_{1}-v_{1}^{*}\right)^{2}+\left(v_{2}-v_{2}^{*}\right)^{2}+\left(v_{3}-v_{3}^{*}\right)^{2}\right] / 3}$, where $v_{i}^{*}$ is the reference price and $v_{i}$ is the computed price at $x_{i}=\{90,100,110\}$. The priori chosen convex combination parameters are $c=\{0,0.5,1\}$.

Table II, Table III and Table IV show the results when $c=\{0,0.5,1\}$ respectively.

TABLE II. Numerical results when $\mathrm{c}=0$.

\begin{tabular}{cccccc|}
\hline $\mathrm{n}$ & $\mathrm{m}$ & Error at 90 & Error at 100 & Error at 110 & RMSE \\
\hline 200 & 80 & $4.611 \mathrm{e}-4$ & $-3.140 \mathrm{e}-2$ & $-5.176 \mathrm{e}-3$ & 0.01837 \\
400 & 160 & $7.738 \mathrm{e}-4$ & $-8.975 \mathrm{e}-3$ & $-1.303 \mathrm{e}-3$ & 0.005255 \\
800 & 320 & $5.475 \mathrm{e}-4$ & $-2.843 \mathrm{e}-3$ & $-3.138 \mathrm{e}-4$ & 0.001681 \\
1600 & 640 & $3.157 \mathrm{e}-4$ & $-1.013 \mathrm{e}-3$ & $-7.130 \mathrm{e}-5$ & 0.0006439 \\
\hline
\end{tabular}

TABLE III. NUMERICAL RESULTS WHEN c $=0.5$.

\begin{tabular}{cccccc|}
\hline $\mathrm{n}$ & $\mathrm{m}$ & Error at 90 & Error at 100 & Error at 110 & RMSE \\
\hline 200 & 80 & $2.631 \mathrm{e}-3$ & $-4.686 \mathrm{e}-2$ & $-1.414 \mathrm{e}-2$ & 0.02830 \\
400 & 160 & $1.794 \mathrm{e}-3$ & $-1.661 \mathrm{e}-2$ & $-5.830 \mathrm{e}-3$ & 0.01021 \\
800 & 320 & $1.047 \mathrm{e}-3$ & $-6.648 \mathrm{e}-3$ & $-2.583 \mathrm{e}-3$ & 0.004161 \\
1600 & 640 & $5.640 \mathrm{e}-4$ & $-2.913 \mathrm{e}-3$ & $-1.072 \mathrm{e}-3$ & 0.001821 \\
\hline
\end{tabular}

TABLE IV. NUMERICAL RESULTS WHEN C=1.

\begin{tabular}{cccccc|}
\hline $\mathrm{n}$ & $\mathrm{m}$ & Error at 90 & Error at 100 & Error at 110 & RMSE \\
\hline 200 & 80 & $4.877 \mathrm{e}-3$ & $-6.245 \mathrm{e}-2$ & $-2.311 \mathrm{e}-2$ & 0.03854 \\
400 & 160 & $2.833 \mathrm{e}-3$ & $-2.428 \mathrm{e}-2$ & $-1.035 \mathrm{e}-2$ & 0.01532 \\
800 & 320 & $1.552 \mathrm{e}-3$ & $-1.046 \mathrm{e}-2$ & $-4.853 \mathrm{e}-3$ & 0.006717 \\
1600 & 640 & $8.134 \mathrm{e}-4$ & $-4.815 \mathrm{e}-3$ & $-2.343 \mathrm{e}-3$ & 0.003127 \\
\hline
\end{tabular}

\section{Conclusions}

We considered the accuracy and conditional stability of IMEX-MCNAB scheme applied to the PIDE (2) with varying convex combinations of the zeroth-order term $\lambda v$. This scheme was studied through Fourier stability analysis, and it is found that, it is conditionally stable under Kou's model for all $\lambda \Delta \tau<1 / 2$ and $c \in[0,1]$. The scheme had the best accuracy in our numerical experiments when $c=0$. Thus, we recommend the IMEX-MCNAB scheme with $c=0$ for European options pricing under Kou's jump-diffusion models.

\section{Acknowledgment}

The work is supported by National Natural Science Foundation of China (No. 11571365). Corresponding author: Zuoliang Xu.

\section{References}

[1] F. Black and M. Scholes, The pricing of options and corporate liabilities, [J]. Policitical Economy. 81, 637-659(1973).

[2] R. Merton, Option pricing when underlying stock returns are discontinuous, Journal of Financial Economics. 3, 125-144(1976). 
[3] S.G. Kou, A jump-diffusion model for option pricing, Management Scienece. 48, 10861101(2002).

[4] P. Carr, H. Geman, D.B. Madan, and M. Yor, The fine structure of asset returns: an empirical investigation, Journal of Business. 75, 305-332(2002).

[5] L. Andersen, J. Andreasen, Jump-diffusion processes: volatility smile fitting and numerical methods for option pricing, Rev. Derivatives Research. 4, 231-262(2000).

[6] P.A. Forsyth, Y. d'Halluin and K. R. Vetzal, Robust Numerical Methods for Contingent Claims under Jump-Diffusion Processes, IMA J. Numer. Anal. 25, 87-112(2005).

[7] D. Tavella, C. Randall, Pricing Financial Instruments: The Finite Difference Method, John Wiley $\$ \backslash \& \$$ Sons, Chichester (2000).

[8] S. Salmi, J. Toivanen, An iterative method for pricing American options under jump-diffusion models, Appl. Numer. Math. 61, 821-831(2011).

[9] U. Ascher, S. Ruuth and B. Wetton, Implicit-explicit methods for time-dependent partial differential equations, SIAM J. Numer. Anal. 32, 797-823(1995).

[10] J. Frank, W. Hundsdorfer and J. Verwer, On the stability of implicit-explicit linear multistep methods, Appl. Numer. Math. 25, 193-205(1997).

[11] S. Salmi, J. Toivanen, IMEX schemes for pricing options under jump-diffusion models, Applied Numerical Mathematics. 84, 33-45(2014).

[12] W. Hundsdorfer, S.J. Ruuth, IMEX extensions of linear multistep methods with general monotonicity and boundedness properties, J. Comput. Phys. 225, 2016-2042(2007).

[13] J. Toivanen, Numerical valuation of European and American options under Kou's jumpdiffusion model, SIAM J. Sci. Comput. 30, 1949-1970(2008). 